\title{
High-affinity ammonium transport by Arabidopsis thaliana AMT1;4
}

\author{
Nino Bindel ${ }^{1} \cdot$ Benjamin Neuhäuser $^{1}(1)$
}

Received: 14 February 2020 / Revised: 1 March 2021 / Accepted: 15 March 2021 / Published online: 30 March 2021

(c) The Author(s) 2021

\begin{abstract}
In plants high affinity transport proteins mediate the essential transport of ammonium across membranes. In Arabidopsis thaliana six of these AMmonium Transporters (AMTs) are encoded by the genome. All of them show a unique expression pattern. While most AMTs are highly expressed in the root, AtAMT1;4 expression is limited to the pollen grains and the pollen tube. Here, we addressed the transport characteristics of AtAMT1;4 in the heterologous Xenopus laevis oocytes system. The transport saturated and showed high affinity for ammonium with a $K_{m}$ value lower than $10 \mu \mathrm{M}$. Based on our electrophysiological analysis, we classified AtAMT1;4 as a high affinity ammonium transporter.
\end{abstract}

Keywords Ammonium transport $\cdot$ High affinity $\cdot$ AMT $\cdot$ MEP $\cdot$ Electrophysiology

\section{Introduction}

To meet the requirements for fast and sufficient nitrogen uptake and distribution within the organism, several distinct transport proteins for ammonium have evolved in the plant Arabidopsis thaliana (Neuhäuser et al. 2007). Six of these AMmonium Transporters (AMTs) are encoded by the Arabidopsis genome, all of them showing diverse localization pointing to specialized functions in the process of ammonium distribution throughout the plant (Yuan 2007). While AtAMT1;5 expression and activity is negligible, three of the high-affinity AMT1 transporters are mainly located in the roots to mediate the transport of ammonium from the soil to the vasculature (Neuhäuser et al. 2007; Yuan et al. 2007). AtAMT1;1 and AtAMT1;3 are located in the plasma membrane of the epidermal and cortical cell layer of the root (Mayer and Ludewig 2006; Loqué et al. 2006). AtAMT1;2 localization is restricted to the cortical and endodermal root cells (Neuhäuser et al. 2007). The localization and high affinity of AtAMT1;1 (In oocytes: $K_{m}=2.7 \mu \mathrm{M}$ (Mayer and Ludewig 2006); in plants: $K_{m}=50.0 \mu \mathrm{M}$ (Yuan et al. 2007)) and AtAMT1;3 (In oocytes: $K_{m}=129 \mu \mathrm{M}(\mathrm{Wu}$

\section{Communicated by E. Schleiff.}

Benjamin Neuhäuser

benjamin.neuhaeuser@uni-hohenheim.de

1 Institute of Crop Science, Nutritional Crop Physiology, University of Hohenheim, Fruwirthstr. 20, 70593 Stuttgart, Germany et al. 2019); in plants: $K_{m}=60.5 \mu \mathrm{M}$ (Yuan et al. 2007)) suggest a function of these two transporters in the direct primary ammonium uptake from the soil. Further transport of ammonium into the vascular tissue depends on transfer across the Casparian strip. AtAMT1;2 with lower affinity but localization in the endodermis was proposed to mediate the uptake into the endodermal cell layer, where the apoplastic flow is blocked by the Casparian strip (Neuhäuser et al. 2007). Indeed it was shown that nitrogen allocation to the shoot is AtAMT1;2 dependent (Duan et al. 2018).

AtAMT2, the sole member of the AMT2 subfamily in Arabidopsis, is strongly expressed in the root as well. While expression in the young root parts can be seen in the trichoblasts and root-hair (Neuhäuser et al. 2009), localization in the older root parts shifts to a preferential expression in the pericycle (Giehl et al. 2017). Expression in the trichoblasts implies a function in primary ammonium uptake while expression in the pericycle was connected with a function in long-distance ammonium transfer to the shoot (Neuhäuser et al. 2009; Giehl et al. 2017).

In strong contrast to these transporters, AtAMT1;4 expression is exclusively found in the pollen grains and the pollen tube (Yuan et al. 2009). This localization excludes a function in primary ammonium uptake. Next to AtAMT1;4 several transporters for organic nitrogen forms are highly expressed in the pollen, suggesting their role in nitrogen loading into the pollen (Bock et al. 2006; Lee and Tegeder 2004). 
The general ammonium transport function of AtAMT1;4 was previously shown by complementation of an ammonium transporter deficient yeast. Further tissue independent overexpression of AtAMT1;4 conferred high-affinity ammonium uptake capacity to uptake deficient Arabidopsis plants (Yuan et al. 2009).

In this work we readdressed the ammonium transport function of AtAMT1;4 in the background free Xenopus leavis oocyte system and compared AtAMT1;4 with AtAMT1;1 mediated transport. Ammonia transport by AtAMT1;4 was proton-coupled and elicited strong inward currents. The net ammonium transport by AtAMT1;4 saturated with a strongly potential dependent affinity.

\section{Results and discussion}

Like the other AtAMT1 transporters, which are involved in high-affinity primary ammonium uptake into the root, the pollen-localized AtAMT1;4 mediates electrogenic ammonium transport when subjected to $300 \mu \mathrm{M}$ ammonium chloride. Ammonium induced strong inward currents with a reversal potential between $+60 \mathrm{mV}$ and $+80 \mathrm{mV}$ (Fig. 1). Normally ammonium currents are expected to reduce towards zero at very positive voltages. Due to the high external ammonium supply, no change in transport direction was expected in all tested voltages. The reversal potential between 50 and $100 \mathrm{mV}$ indicates activation of endogenous chloride channels mediating chloride influx into the oocyte. The activity of these endogenous chloride channels is batchdependent and increases with the age of the oocyte.
In non-injected or water-injected controls ammonium did not elicit ionic inward currents. Both transporters elicited ammonium concentration-dependent currents (Fig. 2a/c). Those currents saturated in a membrane potential-dependent manner. While half-maximal currents of AtAMT1;1 at $-120 \mathrm{mV}$ were reached at $\mathrm{K}_{\mathrm{m}-120 \mathrm{mV}}=16 \mu \mathrm{M}$, AtAMT1;4 mediated currents saturated with $\mathrm{K}_{\mathrm{m}-120 \mathrm{mV}}=6.5 \mu \mathrm{M}$. For AtAMT1;1 this high affinity is easily explained since plants are always competing for sufficient ammonium uptake from the soil with other plants or microorganisms. High ammonium affinity of AtAMT1;1 was shown before (Mayer and Ludewig 2006) but here much higher currents were induced by ammonium (Fig. 1, 2). Previous recordings of AtAMT1;1 mediated ammonium-dependent currents showed high diversity in maximum currents reaching from about $30 \mathrm{nA}$ when oocytes were supplied with $300 \mu \mathrm{M}$ ammonium (Mayer and Ludewig 2006) to about $150 \mathrm{nA}$ when oocytes were supplied with $100 \mu \mathrm{M}$ ammonium (Wood et al. 2006). Maximum currents strongly depend on the amount and stability of the cRNA as well as the oocyte batch. The ammonium currents reported here were stable and reached about $400 \mathrm{nA}$ when supplied with $300 \mu \mathrm{M}$ ammonium (Fig. 1). AtAMT1;4 mediated currents were stable and saturated in a membrane potential-dependent manner, as well. AtAMT1;4 exhibited high ammonium affinity which was superior to the affinity of AtAMT1;1 (Fig. 2). Similarly, low $K_{m}$ values for AtAMT1;4 had been shown by ectopic expression in roots of an ammonium transporter deficient Arabidopsis line (Yuan et al. 2009).

The affinity for ammonium was strongly potential dependent. $K_{m}$ values increased at less negative membrane potential. The $\delta$ value was almost similar for both
Fig. 1 Mean current/voltage relationship of the AtAMT1;1 and AtAMT1;4 mediated currents elicited by $300 \mu \mathrm{M}$ ammonium. Oocytes expressing AtAMT1;1 or AtAMT1;4 were subjected to recording solution containing $300 \mu \mathrm{M}$ ammonium or washing solution without ammonium. To yield ammonium-dependent currents, the currents without ammonium were subtracted from currents with ammonium. Both proteins mediated stable inward-directed currents that decreased with decreasing membrane potential. Data are given as means $( \pm S E)$, $n=4$

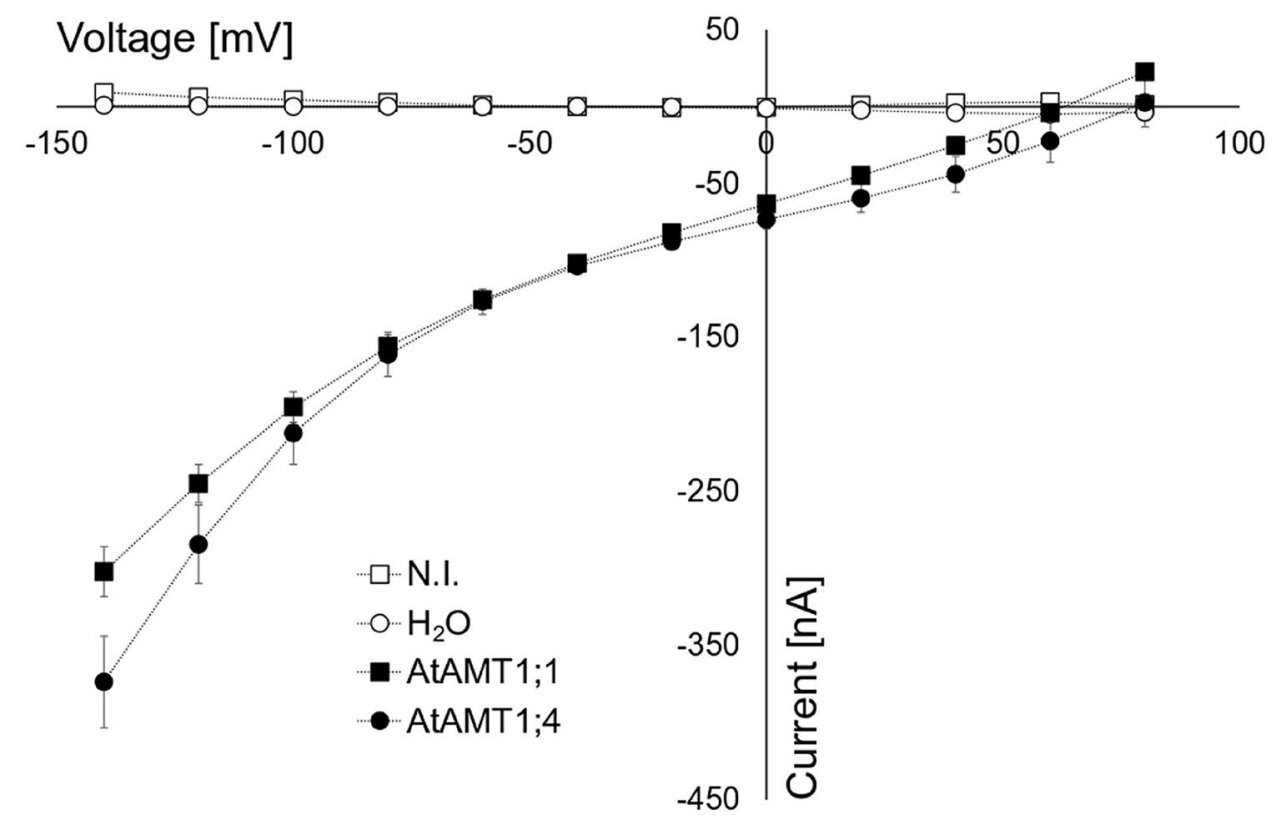



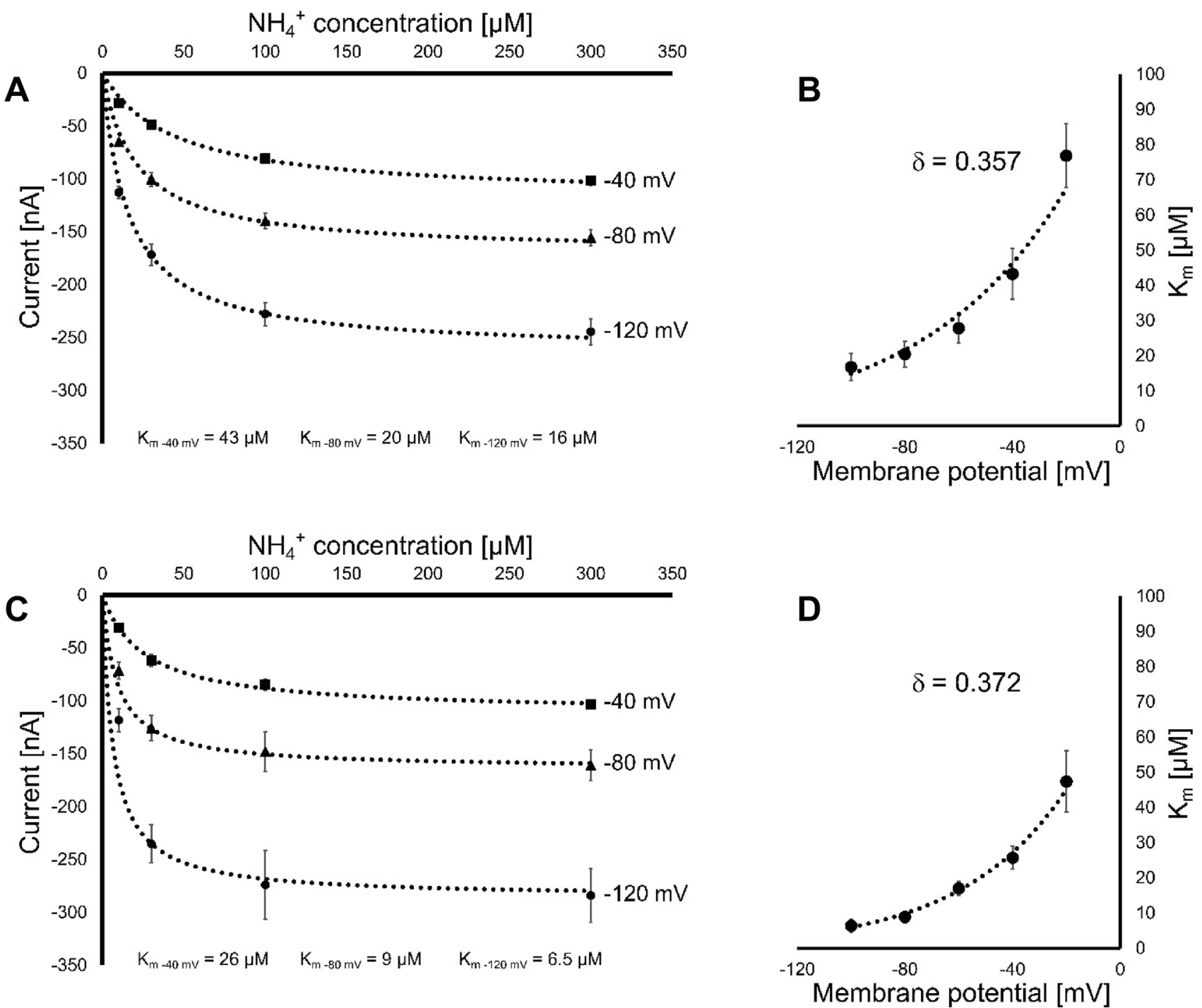

Fig. 2 Transport kinetics of AtAMT1;1 and AtAMT1;4. When expressed in oocytes both transporters elicited ammonium-dependent currents [(a) AtAMT1;1 and (c) AtAMT1;4]. The currents saturated at increasing concentrations. This transport kinetics were strongly membrane potential-dependent and affinity increased $\left(\mathrm{K}_{\mathrm{m}}\right.$ decreased)

transporters with $\delta_{\text {AtAMT1;1 }}=0.357$ and $\delta_{\text {AtAMT } 1 ; 4}=0.372$ (Fig. 2b/d). Therefore, ammonium might be recruited to a binding-side approximately $37 \%$ inside the membrane electric field and then deprotonated during the further transport process.

Binding of ammonium followed by deprotonation was proposed by molecular dynamic transport simulation based on available protein structures (Javelle et al. 2008; Nygaard et al. 2006; Zheng et al. 2004; Khademi et al. 2004) and has recently been suggested to be a general feature of proteins from the AMT (AMmonium Transporter)/ Mep (Methylammonium Permease)/] Rh (Rhesus) family (Ariz et al. 2018). Based on electrophysiologic data, a binding with decreasing membrane potential [(b) AtAMT1;1 and (d) AtAMT1;4]. $\mathbf{a}$ and $\mathbf{c}$ : data are given as means $( \pm \mathrm{SE}), n \geq 4$; (b and d) : data is given as means $( \pm \mathrm{SD}), n \geq 4$. a and $\mathbf{c}$ : dotted lines give fits to Michaelis-Menten kinetics; (b and d) dotted lines give fits to the equation given in experimental procedures

side located deeply inside the transporter pore is in accordance with calculations for other plant AMT1 proteins (Neuhäuser et al. 2007; Straub et al. 2014). In AMT/Mep/Rh proteins a phenylalanine gate blocking the open pore and preventing further uncontrolled ion flux (Ganz et al. 2019) is located approximately $40 \%$ inside the membrane electric field (Zheng et al. 2004; Khademi et al. 2004; Ullmann et al. 2012). These Phenylalanine residues are highly conserved in AMT/MEP/Rh proteins and are directly involved in blocking ammonium ion flux through the central pore but do not seem to be involved in substrate deprotonation (Ganz et al. 2019). Deprotonation might take place at an ensuing twin-Histidine 
motif (Ullmann et al. 2012; Ganz et al. 2020; Lamoureux et al. 2010).

The physiologic function of this high-affinity net ammonium transport by AtAMT1;4 can only be speculated on. The expression in young microspores suggests that ammonium transport is needed to supply the microspore with nitrogen for its further development (Yuan et al. 2009). Still, no obvious phenotype was observed for the knockout mutant in Arabidopsis thaliana (Yuan et al. 2009). Therefore, the significance of AtAMT1;4 might be restricted to specific conditions or other transporters e.g. for organic nitrogen might compensate the loss of ammonium transport by AtAMT1;4. The high substrate affinity of AtAMT1;4 allows us to speculate that free ammonium concentrations in the anther tissue surrounding the pollen are minor.

We here report the first quantitative transport analysis of AtAMT1;4 in a background-free heterologous system. We confirmed high-affinity net ammonium transport by AtAMT1;1 and classified AtAMT1;4 as a high-affinity transporter mediating net ammonium transport.

\section{Experimental procedures}

\section{Construct preparation}

The coding sequence of AtAMT1;4 (At4g28700) was amplified from genomic Col-0 DNA. Primer sequences for amplification included restriction enzyme cut sites for XbaI and NcoI. (Primer sequences: AtAMT1;4-XbaI-Fw: ATAtctagaATGGCGTCGGCTCTCTCTT AtAMT1;4-NcoI-Rv: ATTccatggTCAAACACCTACATTGGGATCATTATC) The PCR product, as well as the $\mathrm{pOO} 2$ vector, were both $\mathrm{XbaI} /$ NcoI digested, cleaned by gel-electrophorese and T4 ligated. Correct insertion of the AtAMT1;4 CDS into pOO2 was tested by Sanger-sequencing. AtAMT1;1-pOO2 was present in the lab (Mayer and Ludewig 2006).

\section{Preparation of cRNA and injection of oocytes}

Dissected and preselected Xenopus laevis oocytes were obtained from Ecocyte Bioscience (Dortmund, Germany). Oocytes were stored in ND96 solution $(96 \mathrm{mM} \mathrm{NaCl}$, $2 \mathrm{mM} \mathrm{KCl}, 1 \mathrm{mM} \mathrm{MgCl} 2,1.8 \mathrm{mM} \mathrm{CaCl}_{2}, 2.5 \mathrm{mM}$ sodium pyruvate, $5 \mathrm{mM}$ HEPES adjusted to $\mathrm{pH}$ of 7.4 by $\mathrm{NaOH}$ ) at $4{ }^{\circ} \mathrm{C}$ prior to injection. cRNA was produced from MluI linearized and phenol-chloroform purified $\mathrm{pOO} 2$ plasmids containing the CDS of AtAMT1;1 or AtAMT1;4 using the mMESSAGE mMACHINETM SP6 Transcription Kit (Life Technologies GmbH, Darmstadt; Germany) following the manufactures instructions. $50 \mathrm{nl}$ cRNA with a concentration of $400 \mathrm{ng} / \mu \mathrm{l}$ were injected into each oocyte. Oocytes were kept in ND96 for 3 days at $18{ }^{\circ} \mathrm{C}$ before electrophysiological measurements were performed.

\section{Electrophysiological measurements}

Electrophysiology was performed in a small recording chamber (as described before (Mayer and Ludewig 2006)) containing Choline-Cl recording solution $(110 \mathrm{mM}$ choline chloride, $2 \mathrm{mM} \mathrm{CaCl}$, $2 \mathrm{mM} \mathrm{MgCl}_{2}, 5 \mathrm{mM}$ MES, $\mathrm{pH}$ adjusted to 5.5 with Tris). Variable ammonium concentrations were added as $\mathrm{NH}_{4} \mathrm{Cl}$ salt. Currents without added ammonium were subtracted at each voltage. The concentration dependence of currents was fitted using the following equation: $I=I_{\max } /\left(1+K_{m} / \mathrm{c}\right)$, with $I_{\max }$ (maximal current at saturating concentration), $K_{m}$ (substrate concentration permitting half-maximal currents), c (experimentally used ammonium concentration). The voltage dependence $\delta$ of the $\mathrm{K}_{\mathrm{m}}$ was calculated using the following equation: $K_{m}(\delta)=K_{m(0 \mathrm{mV})} \mathrm{X} \exp (\delta \mathrm{X} e \mathrm{X} \mathrm{V} / k \mathrm{X} \mathrm{T})$, with $\delta($ fractional electrical distance), $e$ (elementary charge), $\mathrm{V}$ (membrane potential), $k$ (Boltzmann's constant), and T (absolute temperature).

\section{Author contribution statement}

N.B. and B.N. performed the experiments. B.N. analysed the data. B.N. planned the experiments and wrote the manuscript. B.N. is the corresponding author.

Acknowledgements We thank Uwe Ludewig for the discussion. An earlier version of this manuscript is available in form of a preprint on the bioRxiv preprint server for biology: Bindel, N. and Neuhäuser, B. (2019) Electrophysiological characterization of AtAMT1;4, an extraordinarily high-affinity ammonium transporter from Arabidopsis thaliana. (https://doi.org/10.1101/820712).

Funding Open Access funding enabled and organized by Projekt DEAL. Parts of this project were funded by the Federal Ministry of Education and Research (01PL16003) and the Deutsche Forschungsgemeinschaft (NE 1727/2-2). The responsibility for the content lies with the corresponding author.

Data availability All data are part of the manuscript.

\section{Declarations}

Conflict of interests The authors declare no conflict of interests.

Open Access This article is licensed under a Creative Commons Attribution 4.0 International License, which permits use, sharing, adaptation, distribution and reproduction in any medium or format, as long as you give appropriate credit to the original author(s) and the source, provide a link to the Creative Commons licence, and indicate if changes were made. The images or other third party material in this article are included in the article's Creative Commons licence, unless indicated 
otherwise in a credit line to the material. If material is not included in the article's Creative Commons licence and your intended use is not permitted by statutory regulation or exceeds the permitted use, you will need to obtain permission directly from the copyright holder. To view a copy of this licence, visit http://creativecommons.org/licenses/by/4.0/.

\section{References}

Ariz I et al (2018) Nitrogen isotope signature evidences ammonium deprotonation as a common transport mechanism for the AMTMep-Rh protein superfamily. Sci Adv 4:eaar3599

Bock KW et al (2006) Integrating membrane transport with male gametophyte development and function through transcriptomics. Plant Physiol 140:1151-1168

Duan F, Giehl RFH, Geldner N, Salt DE, von Wirén N (2018) Root zone-specific localization of AMTs determines ammonium transport pathways and nitrogen allocation to shoots. PLOS Biol 16:e2006024

Ganz P et al (2019) A pore-occluding phenylalanine gate prevents ion slippage through plant ammonium transporters. Sci Rep 9:16765

Ganz P et al (2020) A twin histidine motif is the core structure for highaffinity substrate selection in plant ammonium transporters. J Biol Chem. https://doi.org/10.1074/jbc.RA119.010891

Giehl RFH et al (2017) A Critical Role of AMT2;1 in Root-toshoot translocation of ammonium in Arabidopsis. Mol Plant 10:1449-1460

Javelle A et al (2008) Substrate binding, deprotonation, and selectivity at the periplasmic entrance of the Escherichia coli ammonia channel AmtB. Proc Natl Acad Sci USA 105:5040-5045

Khademi S et al (2004) Mechanism of ammonia transport by Amt/ MEP/Rh: structure of AmtB at 1.35 A. Science 305:1587-1594

Lamoureux G, Javelle A, Baday S, Wang S, Bernèche S (2010) Transport mechanisms in the ammonium transporter family. Transfus Clin Biol 17:168-175

Lee Y-H, Tegeder M (2004) Selective expression of a novel highaffinity transport system for acidic and neutral amino acids in the tapetum cells of Arabidopsis flowers. Plant J 40:60-74

Loqué D et al (2006) Additive contribution of AMT1;1 and AMT1;3 to high-affinity ammonium uptake across the plasma membrane of nitrogen-deficient Arabidopsis roots. Plant J 48:522-534
Mayer M, Ludewig U (2006) Role of AMT1;1 in NH4+ acquisition in Arabidopsis thaliana. Plant Biol (Stuttg) 8:522-528

Neuhäuser B, Dynowski M, Mayer M, Ludewig U (2007) Regulation of $\mathrm{NH} 4+$ transport by essential cross talk between AMT monomers through the carboxyl tails. Plant Physiol 143:1651-1659

Neuhäuser B, Dynowski M, Ludewig U (2009) Channel-like NH3 flux by ammonium transporter AtAMT2. FEBS Lett 583:2833-2838

Nygaard TP, Rovira C, Peters GH, Jensen MØ (2006) Ammonium recruitment and ammonia transport by $E$. coli ammonia channel AmtB. Biophys J 91:4401-4412

Straub D, Ludewig U, Neuhäuser B (2014) A nitrogen-dependent switch in the high affinity ammonium transport in Medicago truncatula. Plant Mol Biol 86:485-494

Ullmann RT, Andrade SLA, Ullmann GM (2012) Thermodynamics of transport through the ammonium transporter Amt-1 investigated with free energy calculations. J Phys Chem B 116:9690-9703

Wood CC, Porée F, Dreyer I, Koehler GJ, Udvardi MK (2006) Mechanisms of ammonium transport, accumulation, and retention in ooyctes and yeast cells expressing Arabidopsis AtAMT1;1. FEBS Lett 580:3931-3936

Wu X et al (2019) Ammonium and nitrate regulate NH4+ uptake activity of Arabidopsis ammonium transporter AtAMT1;3 via phosphorylation at multiple C-terminal sites. J Exp Bot 70:4919-4930

Yuan L et al (2007) The organization of high-affinity ammonium uptake in Arabidopsis roots depends on the spatial arrangement and biochemical properties of AMT1-type transporters. Plant Cell 19:2636-2652

Yuan L et al (2009) AtAMT1;4, a pollen-specific high-affinity ammonium transporter of the plasma membrane in Arabidopsis. Plant Cell Physiol 50:13-25

Zheng L et al (2004) The mechanism of ammonia transport based on the crystal structure of AmtB of Escherichia coli. Proc Natl Acad Sci USA 101:17090-17095

Publisher's Note Springer Nature remains neutral with regard to jurisdictional claims in published maps and institutional affiliations. 